\title{
Plasma concentrations of osteopontin, but not thrombin-cleaved osteopontin, are associated with the presence and severity of nephropathy and coronary artery disease in patients with type 2 diabetes mellitus
}

Xiaoxiang Yan ${ }^{1,2,3}$, Motoaki Sano ${ }^{3}$, Lin Lu ${ }^{1,2}$, Wei Wang ${ }^{2}$, Qi Zhang ${ }^{1}$, Ruiyan Zhang ${ }^{1}$, Lingjie Wang ${ }^{2}$, Qiujing Chen², Keiichi Fukuda ${ }^{3}$, Weifeng Shen ${ }^{1,2^{*}}$

\begin{abstract}
Background: The aim of the present cross-sectional study was to assess possible associations between osteopontin (OPN), and thrombin-cleaved (N-half) OPN, and nephropathy and coronary artery disease (CAD) in patients with type 2 diabetes mellitus (T2DM).

Methods: Plasma levels of OPN, N-half OPN, and high-sensitivity C-reactive protein (hsCRP) were determined in 301 diabetic patients with $(n=226)$ or without $(n=75)$ angiographically documented CAD (luminal diameter narrowing $>50 \%$ ), as well as in 75 non-diabetic controls with normal angiography. The estimated glomerular filtration rate (eGFR) was calculated in all patients.
\end{abstract}

Results: Plasma levels of OPN and hsCRP were significantly higher in patients with T2DM compared with controls. In addition, there was a higher occurrence of moderate renal insufficiency and lower eGFR in patients with T2DM (all $P<0.01$ ). T2DM patients in whom OPN levels were greater than the median value had higher serum creatinine levels, a greater prevalence of mild or moderate renal insufficiency, a higher incidence of CAD, and lower eGFR (all $P<0.05)$ than T2DM patients in whom OPN levels were the same as or lower than the median value. However, there were no differences in these parameters when patients were stratified according to plasma N-half OPN levels. Furthermore, there was a significant correlation between OPN, but not N-half OPN, and the severity of nephropathy and CAD in diabetes. After adjustment for potential confounders and treatments, multiple linear regression analysis demonstrated an independent association between OPN, but not N-half OPN, and eGFR. Multivariate logistic regression revealed that higher OPN levels conferred a fourfold greater risk of renal insufficiency and CAD in patients with T2DM.

Conclusions: The results of the present study demonstrate that there is an independent association between plasma levels of OPN, but not N-half OPN, and the presence and severity of nephropathy and CAD in diabetes.

\section{Background}

Micro- and macro-vasculopathies, such as nephropathy and coronary artery disease $(\mathrm{CAD})$, respectively, are common in diabetes and constitute the major causes of

\footnotetext{
* Correspondence: rjshenweifeng@yahoo.com.cn

'Department of Cardiology, Rui Jin Hospital, Jiaotong University School of Medicine, Shanghai 200025, China

Full list of author information is available at the end of the article
}

death for these patients [1,2]. Proinflammatory cytokines play a critical role in the pathogenesis of diabetic complications through various biochemical and cellular pathways [1,3-5]. Full-length osteopontin (OPN), a macrophage chemotactic protein, was originally identified as a mediator involved in bone remodeling, chronic inflammatory and autoimmune diseases [6,7], and subsequently demonstrated to play an important role in the 
development of cardiovascular diseases [6,8]. OPN was synthesized by monocytes/macrophages, endothelial cells, and vascular smooth muscle cells, and proved to express in neointima and calcified atheromatous plaque $[6,9]$. In contrast, an OPN neutralizing antibody could inhibit rat carotid neointimal formation after endothelial denudation [10]. Clinically, a significant association has been demonstrated, independent of traditional risk factors, between plasma OPN levels and atherosclerotic plaque formation in general patients $[11,12]$. Furthermore, OPN expression has been shown to be upregulated in the vascular wall of diabetic patients and diabetic animal models, which might be induced by high glucose and advanced glycation endproduct [9,13-15]. In kedney, OPN has also been identified in glomerular mesangial cells, podocytes, and endothelial cells [16-18]. Microarray analyses of diabetic versus normal kidneys identified OPN as one of the major genes upregulated in humans with diabetic nephropathy and in mice with either type 1 diabetes or the type $2 \mathrm{db} / \mathrm{db}$ model of diabetes [19]. OPN knockout mice were protected from diabetes-induced albuminuria and mesangial expansion [18]. A strong correlation between higher OPN levels and more severe diabetic albuminuria and glomerulosclerosis has been demonstrated in various models of diabetic nephropathy $[19,20]$. All these observations suggested a possible role of OPN in accelerated atherogenesis and the development of renal disease in diabetes mellitus.

Structurally, OPN contains several cell-interacting domains, as well as protease-cleavage sites, that may be important in regulating its activity. These domains include: (i) an arginine-glycine-aspartate (RGD)containing domain that interacts with cell surface integrins $\alpha v \beta 3, \alpha v \beta 1, \alpha v \beta 5$, and $\alpha 8 \beta 1$, promoting the migration and/or growth potential of lymphocytes, macrophages, endothelial cells, and vascular smooth muscle cells; and (ii) a cryptic serine-valine-valinetyrosine-glutamate-leucine-arginine (SVVYGLR)-containing domain cleaved by thrombin that interacts with $\alpha 9 \beta 1, \alpha 4 \beta 1$, and $\alpha 4 \beta 7$ integrins and mediates cell adhesion to the $\mathrm{NH}_{2}$-terminal fragment of OPN, causing inflammation in an RGD-independent manner $[6,21,22]$. Patients with diabetes often have a chronic procoagulant state, as reflected by increased thrombin activity and elevated circulating thrombin/anti-thrombin complexes [23-25]. Thrombin-cleaved (N-half) OPN levels are elevated in the vitreous fluid of patients with diabetic retinopathy [26], as well as in the synovial fluid and plasma of patients with rheumatoid arthritis $[7,27]$, highlighting the possibility that this cytokine may be involved in local inflammation. Because diabetes per se is an inflammatory process with increased cytokine levels and enhanced thrombin activity in the vascular wall $[1,23]$, in the present study we tested the hypothesis that there may be an association between both OPN and N-half OPN levels and the presence and severity of nephropathy and CAD in patients with type 2 diabetes mellitus (T2DM).

\section{Methods}

The study protocol was approved by the Ruijin Hospital ethics committee. Written informed consent was obtained from all patients prior to their inclusion in the study.

\section{Study population}

The present study was performed on 301 consecutive diabetic patients (171 men and 130 women) undergoing angiography for suspected CAD or percutaneous coronary intervention (PCI) between June 2005 and October 2007. Patients with concomitant valvular heart disease, cardiomyopathy, acute renal failure, acute and chronic viral or bacterial infections, asthma, tumors, or connective tissue diseases, and those undergoing coronary artery bypass surgery or on dialysis, were excluded from the study. In addition, patients with acute coronary syndrome, in-stent restenosis or those with symptomatic heart failure were excluded from the study. Patients with type 1 diabetes mellitus were identified on the basis of $\mathrm{C}$-peptide measurements and were also excluded from the study. T2DM was diagnosed using the following criteria: (i) fasting plasma glucose levels $\geq 7.0 \mathrm{mmol} / \mathrm{L}$ on two occasions; (ii) two 2-h postprandial plasma glucose readings $\geq 11.1 \mathrm{mmol} / \mathrm{L}$ after a glucose load of $75 \mathrm{~g}$; (iii) two casual glucose readings $\geq 11.1 \mathrm{mmol} / \mathrm{L}$; or (iv) treatment with oral hypoglycemic drugs or parenteral insulin. The clinical characteristics and risk factors for CAD were recorded for each patient. Almost all T2DM patients were receiving antidiabetic treatment and most were taking statins and angiotensin-converting enzyme (ACE) inhibitors or angiotensin receptor blockers (ARBs).

Seventy-five consecutive non-diabetic patients (38 men, 37 women) who underwent coronary angiography for suspected CAD but had normal coronary arteries served as the control group. The professional activities and diet styles of the control group were matched with those of the diabetic patients. Liver and renal function tests were normal.

To elucidate the relationship between OPN or N-half OPN and nephropathy or CAD in diabetes, T2DM patients were divided into two groups, those with higher than median plasma values of OPN $(235.0 \mathrm{ng} / \mathrm{mL})$ and $\mathrm{N}$-half OPN (25.0 pmol/L) and those with median or lower plasma values of OPN and N-half OPN. This was done because the distribution of plasma values of OPN and half-OPN is not normal. 


\section{Coronary angiography}

Selective coronary angiography was performed using a femoral or radial artery approach by cardiologists blinded to the study protocol. Significant CAD was diagnosed visually if the narrowing of the luminal diameter of a major epicardial coronary artery was $\geq 50 \%$. Patients with significant CAD were further classified into those with one-, two-, or three-vessel disease depending on the number of coronary arteries involved. A $\geq 50 \%$ narrowing of the left main coronary artery was considered as two-vessel disease.

\section{Biochemical investigations}

Blood samples were collected from all patients before coronary angiography and after an overnight fast. Plasma OPN level was determined with a commercially available ELISA kit (Human OPN assay kit, IBL, Gunma, Japan) according to its protocol, which only detects full-length OPN. The sensitivity for OPN was $3.33 \mathrm{ng} / \mathrm{ml}$ with an intra- and inter-assay CV of $<5 \%$ and $<10 \%$, respectively. Plasma levels of $\mathrm{N}$-half OPN were measured using an ELISA kit (Human Osteopontin N-Half Assay Kit, IBL, Gunma, Japan). In brief, 1:2 diluted testing samples were incubated in the N-half OPN antibody pre-coated wells at $37^{\circ} \mathrm{C}$ for $1 \mathrm{~h}$. Following washing, $100 \mathrm{uL}$ of labeled OPN antibody solution was added into each well and incubated for $30 \mathrm{~min}$ at $4^{\circ} \mathrm{C}$. After washing, tetramethyl benzidine was used as a coloring agent, and the absorbance at $450 \mathrm{~nm}$ was measured with an automatic ELISA reader (Bio-Rad, Segrate, Italy). The intra- and interassay coefficients of variation $(\mathrm{CV})$ were $<5 \%$ and $<8 \%$, respectively, with a sensitivity $3.09 \mathrm{pg} / \mathrm{L}$. Furthermore, plasma levels high-sensitivity C-reactive protein (hsCRP) were determined (Quantikine ELISA kits; R\&D Systems, Minneapolis, MN, USA). Estimated glomerular filtration rate (eGFR) was calculated using the formula of the Modification of Diet in Renal Disease study group [26]. Mild, moderate, and severe renal insufficiency was diagnosed when the eGFR was $<90,<60$, and $<30 \mathrm{~mL} / \mathrm{min}$ per $1.73 \mathrm{~m}^{2}$, respectively.

\section{Statistical analysis}

All statistical analyses were performed using SPSS for Windows 13.0 (SPSS Inc., Chicago, IL, USA). Unless indicated otherwise, data are presented as frequencies or percentages for categorical variables and as the mean $( \pm \mathrm{SD})$ or median (interquartile range) for continuous variables. For categorical clinical variables, differences between groups were evaluated with the Chi-squared test. For continuous variables, normal distribution was evaluated with the Kolmolgorov-Smirnov test. Variables that were not normally distributed were log transformed when necessary. Comparisons between groups were made using analysis of variance for variables with a parametric distribution. Correlations were evaluated by calculating Spearman's correlation coefficient. Multiple linear regression analyses with eGFR and OPN as dependent variables were used to assess independent relationships. A multivariate logistic regression model was constructed to assess the independent determinants for a moderate decrease in eGFR, CAD and either of the two in diabetic patients. Two-sided $P \leq 0.05$ was considered significant.

\section{Results}

\section{Clinical characteristics}

Compared with non-diabetic controls, T2DM patients were older, had higher systolic and diastolic blood pressures, higher triglyceride and fasting glucose levels, and lower high-density lipoprotein-cholesterol levels. More diabetic patients were receiving treatment with statins and ACE inhibitors or ARBs than controls (Table 1).

\section{OPN and N-half OPN levels in relation to nephropathy and CAD}

Compared with controls, diabetic patients had significantly higher OPN $(P<0.001)$, hsCRP $(P<0.001)$, and creatinine $(P<0.01)$ levels, were more likely to have moderate renal insufficiency $(P<0.01)$, and had a lower eGFR $(P<0.001$; Table 2$)$.

Serum creatinine and occurrence rate of mild or moderate renal insufficiency were higher in T2DM patients with OPN levels greater than the median compared

Table 1 Clinical characteristics and biochemical assessments of study patients

\begin{tabular}{lcc}
\hline Variable & Control $(\mathbf{n}=\mathbf{7 5})$ & Diabetes $(\mathbf{n}=\mathbf{3 0 1})$ \\
\hline Men/Female (n) & $38 / 37$ & $171 / 130$ \\
Age (years) & $56.7 \pm 10.5$ & $65.5 \pm 9.7^{*}$ \\
Smokers (\%) & $14(18.7)$ & $88(29.2)$ \\
Hypertension (\%) & $6(8.0)$ & $252(83.7)^{*}$ \\
$\quad$ SBP (mmHg) & $122 \pm 15$ & $136 \pm 19^{*}$ \\
$\quad$ DBP (mmHg) & $75 \pm 8$ & $79 \pm 11^{\dagger}$ \\
Hyperlipidemia (\%) & $25(33.3)$ & $196(65.1)^{*}$ \\
$\quad$ Triglyceride (mmol/L) & $1.35 \pm 0.65$ & $2.08 \pm 1.63^{*}$ \\
Total cholestrol (mmol/L) & $4.30 \pm 0.80$ & $4.47 \pm 1.00$ \\
HDL-C (mmol/L) & $1.33 \pm 0.35$ & $1.17 \pm 0.31^{*}$ \\
$\quad$ LDL-C (mmol/L) & $2.49 \pm 0.55$ & $2.55 \pm 0.82$ \\
$\quad$ Lipoprotein (a) (g/L) & $0.23 \pm 0.19$ & $0.25 \pm 0.20$ \\
Fast glucose (mmol/L) & $4.85 \pm 0.63$ & $6.98 \pm 2.56^{*}$ \\
HbA1c (\%) & - & $7.57 \pm 1.35$ \\
ACEl or ARB (\%) & $7(9.3)$ & $234(77.7)^{*}$ \\
Statins (\%) & $17(22.7)$ & $237(78.7)^{*}$ \\
\hline Data are given as & &
\end{tabular}

Data are given as either the number of patients (\%), mean \pm SD. ${ }^{*} P<0.001$, ${ }^{\dagger} P<0.01$ compared with the control group.

Abbreviations: SBP, systolic blood pressure; DBP, diastolic blood pressure; $\mathrm{HDL}-\mathrm{C}$, high-density lipoprotein-cholesterol; LDL-C, low-density lipoproteincholesterol; $\mathrm{HbA1c}$, glycated hemoglobin; $\mathrm{ACEl}$, angiotensin-converting enzyme inhibitor; ARB, angiotensin II receptor blocker. 
Table 2 Osteopontin (OPN) and thrombin-cleaved osteopontin ( $\mathrm{N}$-half OPN) in relation to nephropathy and coronary artery disease

\begin{tabular}{|c|c|c|c|c|c|c|}
\hline Variable & $\begin{array}{l}\text { Control } \\
(n=75)\end{array}$ & $\begin{array}{l}\text { Diabetes } \\
(\mathrm{n}=301)\end{array}$ & $\begin{array}{c}\text { OPN } \\
\leq \text { median } \\
(n=150)\end{array}$ & $\begin{array}{c}\text { OPN } \\
\text { >median } \\
(n=151)\end{array}$ & $\begin{array}{l}\text { N-half OPN } \\
\leq \text { median } \\
(n=150)\end{array}$ & $\begin{array}{l}\text { N-half OPN } \\
>\text { median } \\
(\mathrm{n}=151)\end{array}$ \\
\hline \multicolumn{7}{|l|}{ Renal function parameters } \\
\hline Creatinine $(\mu \mathrm{mol} / \mathrm{L})$ & $74.7 \pm 14.6$ & $84.8 \pm 26.1^{\dagger}$ & $79.2 \pm 19.5$ & $90.3 \pm 30.2^{*}$ & $83.9 \pm 27.3$ & $85.8 \pm 24.8$ \\
\hline $\mathrm{eGFR}\left(\mathrm{mL} / \mathrm{min} / 1.73 \mathrm{~m}^{2}\right)$ & $89.6 \pm 20.3$ & $79.8 \pm 20.9^{*}$ & $84.1 \pm 19.5$ & $75.5 \pm 21.3^{*}$ & $80.9 \pm 20.2$ & $78.6 \pm 21.5$ \\
\hline eGFR $<90 \mathrm{~mL} / \mathrm{min} / 1.73 \mathrm{~m}^{2}(\%)$ & $45(60)$ & $206(71.3)$ & $91(63.6)$ & $115(78.8)^{\dagger}$ & $106(72.6)$ & $100(69.9)$ \\
\hline eGFR $<60 \mathrm{~mL} / \mathrm{min} / 1.73 \mathrm{~m}^{2}(\%)$ & $2(2.7)$ & $48(16.6)^{\dagger}$ & $16(11.2)$ & $32(21.9)^{\ddagger}$ & $19(13.0)$ & $29(20.3)$ \\
\hline CAD (\%) & - & $226(75.1)$ & $104(69.3)$ & $122(80.8)^{\ddagger}$ & $111(74.0)$ & $115(76.2)$ \\
\hline \multicolumn{7}{|l|}{ Inflammatory cytokines } \\
\hline OPN (ng/mL) & $218(190,261)$ & $235(201,284)^{*}$ & $201(186,218)$ & $284(258,359)^{*}$ & $242(212,279)$ & $226(191,286)$ \\
\hline N-half OPN (pmol/L) & $23.8(18.9,41.9)$ & $25.3(20.6,34.9)$ & $27.9(20.8,42.7)$ & $23.8(20.4,31.4)^{\dagger}$ & $20.6(18.7,22.3)$ & $34.8(29.3,44.3)^{*}$ \\
\hline $\mathrm{HsCRP}(\mathrm{ng} / \mathrm{mL})$ & $150(102,330)$ & $286(139,675)^{*}$ & $206(130,373)$ & $426(173,1049)^{*}$ & $285(149,629)$ & $288(130,790)$ \\
\hline
\end{tabular}

Data are given as mean \pm SD or the median (interquartile ranges), as appropriate. ${ }^{*} P<0.001,{ }^{\dagger} P<0.01,{ }^{\ddagger} P<0.05$ compared with the control group, the group with OPN values below the median value and the group with $\mathrm{N}$-half OPN values below the median, respectively.

Abbreviations: eGFR, estimated glomerular filtration rate; CAD, coronary artery disease; OPN, osteopontin; N-half OPN, Thrombin-cleaved N-half osteopontin; hsCRP, high sensitive C-reactive protein.

with those with OPN levels lower than the median (creatinine $90.3 \pm 30.2$ vs. $79.2 \pm 19.5 \mu \mathrm{mol} / \mathrm{L}$, respectively $(P<0.001)$; mild renal insufficiency $78.8 \%$ vs. $63.6 \%$, respectively $(P<0.01)$; and moderate renal insufficiency $21.9 \%$ vs $11.2 \%$, respectively $(P<0.05)$ ); in contrast, eGFR was higher in T2DM patients with OPN levels below the median $(84.1 \pm 19.5$ vs $75.5 \pm 21.3 \mathrm{~mL} /$ min per $1.73 \mathrm{~m}^{2} ; P<0.001$; Table 2). There were no significant differences in creatinine levels, eGFR, and the occurrence of renal insufficiency between groups stratified according to N-half OPN values (Table 2). In addition, there was a negative correlation found between OPN, but not N-half OPN, and eGFR in T2DM patients $(r=-0.258 ; P<0.001$; Figure $1 \mathrm{~A})$, as well as stepwise increases in mean OPN concentrations in patients with deteriorating renal function $(237 \pm 72,268 \pm 120$, and $338 \pm 203 \mathrm{ng} / \mathrm{mL}$ in T2DM patients with normal, mild, moderate renal insufficiency, respectively; $P<0.001$; Figure $1 \mathrm{~B})$. In the present study, the five T2DM patients whose eGFR was $<30 \mathrm{~mL} / \mathrm{min}$ per $1.73 \mathrm{~m}^{2}$ had highest plasma OPN levels $(402 \pm 141 \mathrm{ng} / \mathrm{mL})$. However, there was no difference in the percent of N-half OPN (N-half OPN/OPN*100\%) in T2DM patients with normal, mild and moderate renal insufficiency $(P=0.65)$.

Patients with OPN concentrations greater than the median value had a higher incidence of CAD than those with OPN levels below the medium value $(80.8 \%$ vs. $69.3 \%$, respectively; $P<0.05$ ), but differences were not found for patient groups stratified according to N-half OPN values ( $76.2 \%$ vs. $74.0 \% ; P>0.05$; Table 2$)$. Furthermore, in patients with T2DM, the presence and severity of CAD were significantly associated with plasma OPN $\left(P=0.002\right.$ and $\left.P_{\text {trend }}=0.002\right)$, but not with $\mathrm{N}$-half OPN levels (Figure $1 C, D$ ). In addition, the percent of $\mathrm{N}$-half
OPN was similar in T2DM patients with and without CAD $(P=0.55)$.

In T2DM patients, there was a positive correlation between plasma OPN levels and hsCRP $(r=0.394 ; P<$ 0.001). After adjusting for age, the associations between plasma OPN and hsCRP $(r=0.383 ; P<0.001)$ remained significant. However, $\mathrm{N}$-half OPN did not correlate with other variables in this study.

\section{Multivariate regression analysis on nephropathy and CAD} in diabetes

Multiple linear regression analysis showed that after adjustment for several potential confounding variables in Model A, eGFR was independently correlated with OPN $(P=0.002)$. In a similar analysis, when OPN was used as a dependent variable in Model B after adjustment for several potential confounding variables, independent associations were found between OPN and $\mathrm{N}$-half OPN, hsCRP, and eGFR (all $P<0.01$; Table 3).

Multiple logistic regression analysis revealed that after adjustment for several potential confounding variables, the risk of a moderate reduction in eGFR, the presence of CAD, and a moderate reduction in eGFR or CAD increased more than fourfold $(P=0.006, P=$ 0.034 , and $P=0.027$, respectively) for OPN in the diabetic cohort (Table 4). Furthermore, eGFR was independently associated with the presence of CAD $(P=$ 0.026; Table 4).

\section{Discussion}

The present study demonstrated that plasma levels of OPN, but not N-half OPN, are independently associated with the presence and severity of nephropathy and CAD in diabetes. 

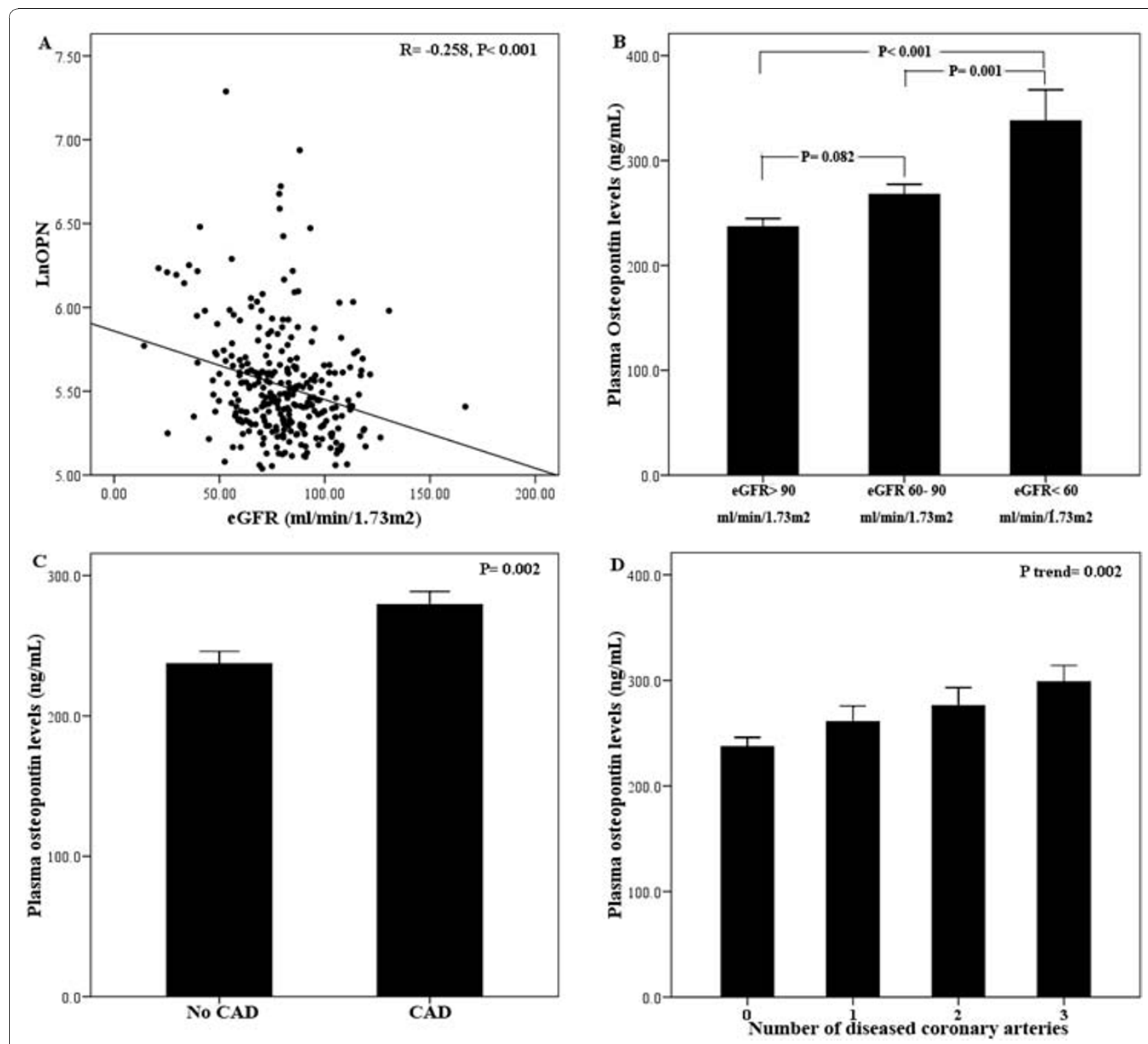

Figure 1 Association between plasma osteopontin (OPN) levels and renal insufficiency and coronary artery (CAD) disease in all diabetic patients. (A) Scatter plot showing the relationship between OPN and estimated glomerular filtration rate (eGFR) in the diabetic population. OPN concentrations were log-transformed and $R$ represents Pearson's correlation coefficient. (B) Plasma OPN levels in patients with different renal function stratified according to eGFR. Data are the mean \pm SEM. Differences among groups were analyzed by one-way ANOVA followed by post hoc analysis (with Bonferroni's correction). (C, D) Relationship between plasma OPN levels and the presence (C) and severity (D) of CAD in diabetes. Data are the mean \pm SEM.

\section{Relationship between plasma OPN levels and nephropathy and CAD in diabetes}

Previous studies have shown that advanced glycation end-products and angiotensin II can stimulate OPN synthesis by a variety of cells, including mesangial cells and podocytes, and initiate local effects of cell spreading, adhesion, and proliferation [28-30]. In rat models of streptozotocin- or high-fat diet-induced diabetic nephropathy, OPN expression is significantly upregulated in the renal cortex and aorta [31,32], and increased OPN expression is strongly correlated with severe diabetic albuminuria and glomerulosclerosis $[19,20]$. In contrast, OPN-knockout mice do not develop albuminuria in response to lipopolysaccharide, and are protected from diabetes-induced mesangial expansion [18]. In patients with diabetic nephropathy, OPN expression is closely related to the degree of cortical interstitial scarring [33]. Yamaguchi et al. found that plasma OPN levels were increased in diabetic patients with renal failure [34]. In the present study, all patients had cardiovascular risk 
Table 3 Multiple linear regression analysis of relationships between selected variables and estimated glomerular filtration rate or osteopontin

\begin{tabular}{lccc}
\hline \multicolumn{1}{l}{ Variable } & $\boldsymbol{\beta}$ & SEM & $\boldsymbol{P}$ \\
\hline Model A: Dependent variable eGFR & & \\
Age & -0.889 & 0.127 & $<0.001$ \\
OPN & -11.967 & 3.823 & 0.002 \\
Model B: Dependent variable OPN & & \\
N-half OPN & -0.136 & 0.027 & $<0.001$ \\
hsCRP & 0.103 & 0.017 & $<0.001$ \\
eGFR & -0.003 & 0.001 & 0.002 \\
\hline
\end{tabular}

In addition to the significant covariables shown in the table, Model A also included sex, cigarette smoking, systolic blood pressure (SBP), total cholesterol, triglyceride, lipoprotein (a), fasting glucose, use of angiotensin receptor blockers (ARBs) or angiotensin-converting enzyme inhibitors (ACEI), use of statins, log-transformed thrombin-cleaved osteopontin (N-half OPN), and log-transformed high-sensitivity C-reactive protein (hsCRP). Model B included sex, age, cigarette smoking, SBP, total cholesterol, triglyceride, lipoprotein (a), fasting glucose, use of ARBs or ACEl, and the use of statins.

factors and had been hospitalized for diagnostic angiography and/or PCI. We found that plasma OPN concentrations were proportional to the severity of renal dysfunction and were an independent risk factor for the presence and severity of renal insufficiency, suggesting that OPN may play a pivotal role in the development of diabetic nephropathy in these high-risk patients. Conversely, the presence of renal insufficiency could lead to elevated plasma OPN concentrations, forming a vicious cycle that exaggerates diabetic nephropathy and atherosclerosis.

Table 4 Determinants for presence of an estimated glomerular filtration rate $<60 \mathrm{~mL} / \mathrm{min}$ per $1.73 \mathrm{~m}^{2}$ and coronary artery disease

\begin{tabular}{cccc}
\hline Variable & Odds ratio & $\mathbf{9 5 \%}$ Confidence interval & $\boldsymbol{P}$ \\
\hline \multicolumn{3}{c}{ Model A: Dependent variable } & moderate reduction in eGFR \\
Age & 1.08 & $1.04-1.14$ & 0.001 \\
OPN & 4.58 & $1.55-13.59$ & 0.006 \\
Model B: Dependent variable CAD & 0.028 \\
Sex & 2.29 & $1.10-4.79$ & $<0.001$ \\
Statins & 5.14 & $2.52-10.48$ & 0.034 \\
OPN & 4.30 & $1.12-16.54$ & 0.026 \\
eGFR & 0.20 & $0.05-0.82$ & 0.002 \\
Model C: Dependent variable moderate reduction in eGFR or CAD \\
Age & 1.06 & $1.02-1.11$ & $<0.001$ \\
Statins & 4.62 & $2.29-9.35$ & 0.027 \\
OPN & 4.49 & $1.18-17.05$ & \\
\hline
\end{tabular}

Models $\mathrm{A}$ and $\mathrm{C}$ also included sex, cigarette smoking, systolic blood pressure (SBP), total cholesterol, triglyceride, lipoprotein (a), fasting glucose, use of angiotensin receptor blockers (ARBs) or angiotensin-converting enzyme inhibitors (ACEI), use of statins, log-transformed thrombin-cleaved osteopontin (N-half OPN), and log-transformed high-sensitivity C-reactive protein (hsCRP). Model $\mathrm{B}$ also included cigarette smoking, SBP, total cholesterol, triglyceride, lipoprotein (a), fasting glucose, use of ARBs or ACEl, log-transformed N-half OPN, log-transformed hsCRP, and log-transformed estimated glomerular filtration rate (eGFR)
Plasma OPN levels have been shown to be correlated with the angiographic severity of CAD, independent of conventional risk factors [12], and have been identified as a potential marker of atherosclerotic disease progression and adverse cardiovascular outcome in patients with stable angina [11]. Consistent with previous observations [35], we found in the present study that OPN was closely associated with hsCRP levels independent of age, suggesting that OPN, as an impaortant inflammatory cytokine, may aggravate the inflammation status in diabetes mellitus. In addition, we demonstrated a significant association between plasma OPN levels and the presence and severity of CAD in diabetic patients, indicating that OPN may be critically involved in the inflammatory processes that take place within the vascular wall in diabetes.

\section{Relationship between plasma concentrations of N-half OPN and nephropathy and CAD in diabetes}

In vitro studies have reported that the $\mathrm{N}$-terminal fragments of OPN generated by both thrombin cleavage and matrix metalloproteinase (MMP) cleavage induce enhanced adhesion compared with the effects of full-length OPN. This appears to be due mostly to increased activity of the RGD site, perhaps an indication of conformational change resulting in higher affinity binding [6]. In a model of rheumatoid arthritis, an antibody specifically neutralizing only the SLAYGLR domain of mouse OPN (homologous to the SVVYGLR of human OPN) greatly abrogated monocyte migration towards the thrombin-cleaved form of OPN and inhibited the proliferation of synovial cells, bone erosion, and inflammatory cell infiltration in arthritic joints [21]. Another study showed that the SVVYGLR sequence induces pro-MMP9 expression in isolated vascular smooth muscle cells, as well as in aortas from diabetic mice [36]. Clinically, N-half OPN levels are elevated in the synovial fluid, urine, and plasma of patients with rheumatoid arthritis $[7,27,35]$, as well as in the vitreous fluid of those with diabetic retinopathy [26], indicating that this cytokine is involved in local inflammation. Because diabetes per se is an inflammatory process with increased cytokine levels and enhanced thrombin activity in the vascular wall [23], we hypothesized that N-half OPN may be associated with the development and severity of nephropathy and CAD in this setting. Unexpectedly, there was no association between N-half OPN and CAD or hsCRP in diabetic patients. This may be due to the relatively lower concentrations of N-half OPN compared with full-length OPN in plasma (the percent of $\mathrm{N}$-half OPN/OPN was no more than 1\%). Alternatively, N-half OPN may exist predominantly in local tissue rather than in the circulation. Further studies are warranted to examine the role of circulating and local vascular N-half OPN in the pathogenesis of diabetic nephropathy and atherosclerosis. 


\section{Clinical relevance of OPN in diabetes}

Type 2 diabetes is an independent risk factor for cardiovascular diseases and a CAD equivalent [2,37]. Its complications mainly include diabetic nephropathy, atherosclerosis and retinopathy [1]. Although hypoglycemic agents can control blood glucose, current treatment is still not sufficient to prevent the development of diabetic complications [37]. Therefore, in these high-risk patients, early detection and effective prevention of the complications become an important issue. In the present study, we found that plasma OPN level, but not N-half OPN parallels with the severity of nephropathy and CAD in diabetes, suggesting that an increased plasma OPN level may be used as an indicator for screening diabetic vasculopathy. Furthermore, since basic research showed that OPN contributes to the development of nephropathy and atherosclerosis in diabetes [10,18-20], inhibition of OPN may become a novel therapeutic strategy for these paitents.

\section{Study limitations}

There are several limitations to the present study. First, the present study was a cross-sectional design; thus, our results reflect only the association between OPN or $\mathrm{N}$-half OPN levels and prevalent rather than incident atherosclerosis. Second, all subjects in the present study were scheduled for coronary angiography and/or PCI, and are thus likely to be more typical of a high-risk patient group than the healthy population at large.

In conclusion, the present study indicates that plasma concentrations of OPN, but not N-half OPN, are independently associated with the presence and severity of nephropathy and CAD in T2DM patients. Approaches involving the inhibition of OPN signaling may prove valuable therapeutic strategies for the prevention of diabetic complications and improved patient outcomes.

\section{Acknowledgements}

This study was supported by a grant from Shanghai Shen Kang High Medical Technology Foundation (to W.F.S.). The first author would like to acknowledge the China Scholarship Council.

\section{Author details}

${ }^{1}$ Department of Cardiology, Rui Jin Hospital, Jiaotong University School of Medicine, Shanghai 200025, China. ${ }^{2}$ Institute of Cardiovascular Diseases, Jiaotong University School of Medicine, Shanghai 200025, China. ${ }^{3}$ Division of Cardiology, Department of Medicine, Keio University School of Medicine, Tokyo 160-8582, Japan.

\section{Authors' contributions \\ $X Y$ contributed to the study design, collected the data, contributed to the discussion, and wrote the manuscript. LL, WW, LW, QZ, RZ, and QC collected the data. MS, KF reviewed/edited the manuscript. WS contributed to the study design, discussion, and reviewed/edited the manuscript. All authors have read and approved the final manuscript.}

\section{Competing interests}

The authors declare that they have no competing interests.
Received: 13 October 2010 Accepted: 29 October 2010

Published: 29 October 2010

\section{References}

1. Orasanu G, Plutzky J: The pathologic continuum of diabetic vascular disease. J Am Coll Cardiol 2009, 53:S35-42.

2. Al-Maskari F, El-Sadig M, Norman JN: The prevalence of macrovascular complications among diabetic patients in the United Arab Emirates. Cardiovasc Diabetol 2007, 6:24.

3. Yan XX, Lu L, Peng WH, Wang LJ, Zhang Q, Zhang RY, Chen QJ, Shen WF: Increased serum HMGB1 level is associated with coronary artery disease in nondiabetic and type 2 diabetic patients. Atherosclerosis 2009, 205:544-548.

4. Rathcke CN, Vestergaard H: YKL-40-an emerging biomarker in cardiovascular disease and diabetes. Cardiovasc Diabetol 2009, 8:61.

5. Pu LJ, Lu L, Xu XW, Zhang RY, Zhang Q, Zhang JS, Hu J, Yang ZK, Ding FH, Chen QJ, Lou S, Shen J, Fang DH, Shen WF: Value of serum glycated albumin and high-sensitivity C-reactive protein levels in the prediction of presence of coronary artery disease in patients with type 2 diabetes. Cardiovasc Diabetol 2006, 5:27.

6. Scatena M, Liaw L, Giachelli CM: Osteopontin: a multifunctional molecule regulating chronic inflammation and vascular disease. Arterioscler Thromb Vasc Biol 2007, 27:2302-2309.

7. Ohshima S, Yamaguchi N, Nishioka K, Mima T, Ishii T, Umeshita-Sasai M, Kobayashi H, Shimizu M, Katada Y, Wakitani S, Murata N, Nomura S, Matsuno H, Katayama R, Kon S, Inobe M, Uede T, Kawase I, Saeki Y: Enhanced local production of osteopontin in rheumatoid joints. J Rheumatol 2002, 29:2061-2067.

8. Okamoto $\mathrm{H}$ : Osteopontin and cardiovascular system. Mol Cell Biochem 2007, 300:1-7.

9. Takemoto M, Yokote K, Nishimura M, Shigematsu T, Hasegawa T, Kon S, Uede T, Matsumoto T, Saito Y, Mori S: Enhanced expression of osteopontin in human diabetic artery and analysis of its functional role in accelerated atherogenesis. Arterioscler Thromb Vasc Biol 2000, 20:624-628.

10. Liaw L, Lombardi DM, Almeida MM, Schwartz SM, deBlois D, Giachelli CM: Neutralizing antibodies directed against osteopontin inhibit rat carotid neointimal thickening after endothelial denudation. Arterioscler Thromb Vasc Biol 1997, 17:188-193.

11. Minoretti P, Falcone C, Calcagnino M, Emanuele E, Buzzi MP, Coen E, Geroldi D: Prognostic significance of plasma osteopontin levels in patients with chronic stable angina. Eur Heart J 2006, 27:802-807.

12. Ohmori R, Momiyama $Y$, Taniguchi $H$, Takahashi R, Kusuhara M, Nakamura $\mathrm{H}$, Ohsuzu F: Plasma osteopontin levels are associated with the presence and extent of coronary artery disease. Atherosclerosis 2003 170:333-337.

13. Nilsson-Berglund LM, Zetterqvist AV, Nilsson-Ohman J, Sigvardsson M, Gonzalez Bosc LV, Smith ML, Salehi A, Agardh E, Fredrikson GN, Agardh CD, Nilsson J, Wamhoff BR, Hultgardh-Nilsson A, Gomez MF: Nuclear factor of activated $T$ cells regulates osteopontin expression in arterial smooth muscle in response to diabetes-induced hyperglycemia. Arterioscler Thromb Vasc Biol 2010, 30:218-224.

14. Takemoto M, Yokote $K$, Yamazaki M, Ridall AL, Butler WT, Matsumoto T, Tamura K, Saito Y, Mori S: Enhanced expression of osteopontin by high glucose in cultured rat aortic smooth muscle cells. Biochem Biophys Res Commun 1999, 258:722-726.

15. Kawamura H, Yokote K, Asaumi S, Kobayashi K, Fujimoto M, Maezawa $Y$, Saito $Y$, Mori S: High glucose-induced upregulation of osteopontin is mediated via Rho/Rho kinase pathway in cultured rat aortic smooth muscle cells. Arterioscler Thromb Vasc Biol 2004, 24:276-281.

16. Nicholas SB, Liu J, Kim J, Ren Y, Collins AR, Nguyen L, Hsueh WA: Critical role for osteopontin in diabetic nephropathy. Kidney Int 2010, 77:588-600.

17. Endlich N, Sunohara M, Nietfeld W, Wolski EW, Schiwek D, Kranzlin B, Gretz N, Kriz W, Eickhoff H, Endlich K: Analysis of differential gene expression in stretched podocytes: osteopontin enhances adaptation of podocytes to mechanical stress. FASEB J 2002, 16:1850-1852.

18. Lorenzen J, Shah R, Biser A, Staicu SA, Niranjan T, Garcia AM, Gruenwald A, Thomas DB, Shatat IF, Supe K, Woroniecki RP, Susztak K: The role of osteopontin in the development of albuminuria. J Am Soc Nephrol 2008, 19:884-890. 
19. Susztak K, Bottinger E, Novetsky A, Liang D, Zhu Y, Ciccone E, Wu D, Dunn S, McCue P, Sharma K: Molecular profiling of diabetic mouse kidney reveals novel genes linked to glomerular disease. Diabetes 2004 53:784-794.

20. Hsieh TJ, Chen R, Zhang SL, Liu F, Brezniceanu ML, Whiteside Cl, Fantus IG, Ingelfinger JR, Hamet $\mathrm{P}$, Chan JS: Upregulation of osteopontin gene expression in diabetic rat proximal tubular cells revealed by microarray profiling. Kidney Int 2006, 69:1005-1015.

21. Yamamoto N, Sakai F, Kon S, Morimoto J, Kimura C, Yamazaki H, Okazaki I, Seki N, Fujii T, Uede T: Essential role of the cryptic epitope SLAYGLR within osteopontin in a murine model of rheumatoid arthritis. J Clin Invest 2003, 112:181-188.

22. Nishimichi N, Higashikawa F, Kinoh HH, Tateishi Y, Matsuda H, Yokosaki Y: Polymeric osteopontin employs integrin alpha9beta1 as a receptor and attracts neutrophils by presenting a de novo binding site. $J$ Biol Chem 2009, 284:14769-14776.

23. Boden G, Vaidyula VR, Homko C, Cheung P, Rao AK: Circulating tissue factor procoagulant activity and thrombin generation in patients with type 2 diabetes: effects of insulin and glucose. J Clin Endocrinol Metab 2007, 92:4352-4358.

24. Jax TW, Peters AJ, Plehn G, Schoebel FC: Hemostatic risk factors in patients with coronary artery disease and type 2 diabetes - a two year follow-up of 243 patients. Cardiovasc Diabetol 2009, 8:48.

25. Jax TW, Peters AJ, Plehn G, Schoebel FC: Relevance of hemostatic risk factors on coronary morphology in patients with diabetes mellitus type 2. Cardiovasc Diabetol 2009, 8:24.

26. Kase S, Yokoi M, Saito W, Furudate N, Ohgami K, Kitamura M, Kitaichi N, Yoshida K, Kase M, Ohno S, Uede T: Increased osteopontin levels in the vitreous of patients with diabetic retinopathy. Ophthalmic Res 2007, 39:143-147.

27. Hasegawa M, Nakoshi Y, lino T, Sudo A, Segawa T, Maeda M, Yoshida T, Uchida A: Thrombin-cleaved osteopontin in synovial fluid of subjects with rheumatoid arthritis. J Rheumatol 2009, 36:240-245

28. Xie Y, Sakatsume M, Nishi S, Narita I, Arakawa M, Gejyo F: Expression, roles, receptors, and regulation of osteopontin in the kidney. Kidney Int 2001, 60:1645-1657.

29. Ren X, Shao H, Wei Q, Sun Z, Liu N: Advanced glycation end-products enhance calcification in vascular smooth muscle cells. J Int Med Res 2009, 37:847-854.

30. Wolak T, Kim H, Ren Y, Kim J, Vaziri ND, Nicholas SB: Osteopontin modulates angiotensin II-induced inflammation, oxidative stress, and fibrosis of the kidney. Kidney Int 2009, 76:32-43.

31. Fischer JW, Tschope C, Reinecke A, Giachelli CM, Unger T: Upregulation of osteopontin expression in renal cortex of streptozotocin-induced diabetic rats is mediated by bradykinin. Diabetes 1998, 47:1512-1518.

32. Towler DA, Bidder M, Latifi T, Coleman T, Semenkovich CF: Diet-induced diabetes activates an osteogenic gene regulatory program in the aortas of low density lipoprotein receptor-deficient mice. J Biol Chem 1998, 273:30427-30434.

33. Junaid A, Amara FM: Osteopontin: correlation with interstitial fibrosis in human diabetic kidney and PI3-kinase-mediated enhancement of expression by glucose in human proximal tubular epithelial cells. Histopathology 2004, 44:136-146.

34. Yamaguchi $H$, Igarashi M, Hirata A, Tsuchiya H, Sugiyama K, Morita $Y$, Jimbu Y, Ohnuma H, Daimon M, Tominaga M, Kato T: Progression of diabetic nephropathy enhances the plasma osteopontin level in type 2 diabetic patients. Endocr J 2004, 51:499-504

35. Shio K, Kobayashi $H$, Asano T, Saito R, Iwadate H, Watanabe H, Sakuma $H$, Segawa T, Maeda M, Ohira H: Thrombin-cleaved osteopontin is increased in urine of patients with rheumatoid arthritis. J Rheumatol 2010, 37:704-710.

36. Lai CF, Seshadri V, Huang K, Shao JS, Cai J, Vattikuti R, Schumacher A, Loewy AP, Denhardt DT, Rittling SR, Towler DA: An osteopontin-NADPH oxidase signaling cascade promotes pro-matrix metalloproteinase 9 activation in aortic mesenchymal cells. Circ Res 2006, 98:1479-1489.

37. Beckman JA, Creager MA, Libby P: Diabetes and atherosclerosis: epidemiology, pathophysiology, and management. JAMA 2002, 287:2570-2581. doi:10.1186/1475-2840-9-70

Cite this article as: Yan et al:: Plasma concentrations of osteopontin, but not thrombin-cleaved osteopontin, are associated with the presence and severity of nephropathy and coronary artery disease in patients with type 2 diabetes mellitus. Cardiovascular Diabetology 2010 9:70.

\section{Submit your next manuscript to BioMed Central and take full advantage of:}

- Convenient online submission

- Thorough peer review

- No space constraints or color figure charges

- Immediate publication on acceptance

- Inclusion in PubMed, CAS, Scopus and Google Scholar

- Research which is freely available for redistribution

Submit your manuscript at www.biomedcentral.com/submit
Ciomed Central 